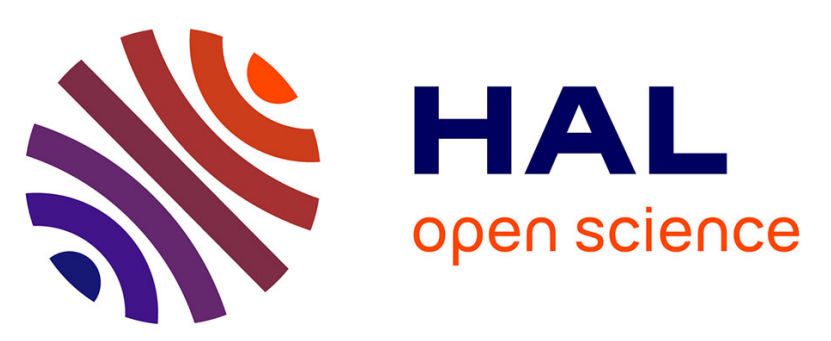

\title{
Lossless progressive compression of meshes for upscaling and upgridding in reservoir simulation with HexaShrink
}

Lauriane Bouard, Laurent Duval, Christophe Preux, Frédéric Payan, Marc

Antonini

\section{- To cite this version:}

Lauriane Bouard, Laurent Duval, Christophe Preux, Frédéric Payan, Marc Antonini. Lossless progressive compression of meshes for upscaling and upgridding in reservoir simulation with HexaShrink. EAGE [European Association of Geoscientists \& Engineers], Jun 2019, London, United Kingdom. hal-02190567

\section{HAL Id: hal-02190567 \\ https://hal-ifp.archives-ouvertes.fr/hal-02190567}

Submitted on 22 Jul 2019

HAL is a multi-disciplinary open access archive for the deposit and dissemination of scientific research documents, whether they are published or not. The documents may come from teaching and research institutions in France or abroad, or from public or private research centers.
L'archive ouverte pluridisciplinaire HAL, est destinée au dépôt et à la diffusion de documents scientifiques de niveau recherche, publiés ou non, émanant des établissements d'enseignement et de recherche français ou étrangers, des laboratoires publics ou privés. 
EAGE, London, UK. 2019

European Association of Geoscientists \& Engineers

\title{
Lossless progressive compression of meshes for upscaling and upgridding in reservoir simulation with HexaShrink
}

\author{
Lauriane Bouard ${ }^{1,3}$, Laurent Duval ${ }^{2}$, Christophe Preux $^{2}$, Frédéric Payan $^{3}$, and Marc Antonini ${ }^{3}$ \\ ${ }^{1}$ IFP Energies nouvelles, Rond-point de l'échangeur de Solaize, 69360 Solaize, France \\ ${ }^{2}$ IFP Energies nouvelles, 1-4 Avenue du Bois Préau, 92852 Rueil-Malmaison, France \\ ${ }^{3}$ Université Côte d'Azur (UCA), CNRS, Laboratoire I3S, 06900 Sophia Antipolis, France
}

2019-07-22

\section{Introduction}

Grids used for reservoir simulation in geosciences are complex structures with large dimensions: until hundreds of thousands cells. Since dip and faulting are common geometric traits in hydrocarbon reservoirs, the corner-point grid $(\mathrm{CPG})$ geometry developed by Ponting (1989) can represent them appropriately. In this geometry, simulation cells are specified by eight corner points, with boundaries arranged along vertical or sloped directions.

Such complex grids are composite objects, combining classically heterogeneous data, such as geometry, cell activity and continuous or categorical properties. They are thus heavy to store, to visualize and to manipulate. Simulations on large meshes are notably time-consuming. To reduce the number of cells, and thus accelerate simulations, several researches have been performed on upscaling methods, to generate coarser grids for specific purposes. However, creating new grids at different resolutions does not solve the data quantity issue.

This study proposes an alternative to such trending "big data" accumulation problems that impact computational performances in experimental and simulation sciences. It relies on HexaShrink, an original decomposition scheme for structured hexahedral volume meshes (Peyrot et al., 2019, 2016). The latter provides a consistent and fully reversible dyadic multiscale representation of mesh geometry and properties. It generates a hierarchy of meshes at increasing levels of details, blending coherently different wavelet-like schemes for each mesh component. By exploiting the similarities across scales, Bouard et al. (2018) recently showed better compressibility of decomposed meshes, when binary files are further compressed with generic lossless coders (gzip, bzip2, LZMA). Indeed, a benchmark of meshes with different size and complexity shows compression ratios of 2.76-13.35, instead of 1.83-12.52 without multiscale decomposition; hence, smaller stored datasets. Additional benefits reside in the coherency of fault networks, with global shape preserved across resolutions, and in the same time offering an access to a cascade of intermediate meshes at reduced resolutions. This approach was initially meant for storage and visualization. Here, we evaluate its consistency with respect to simulation. 
EAGE, London, UK. 2019

European Association of Geoscientists \& Engineers

\section{Method}

The proposed methodology consists in 1) constructing a representative simulation model, 2) applying HexaShrink decomposition on all its components, 3) evaluating the simulation performance on the resulting reduced resolution grids.

The multiscale decompositions used in HexaShrink are based on the broad concept of wavelet transforms (Mallat, 2009). Data is split into a dyadic scheme: approximations at a lower scale, capturing the regularity of the information, and details, embedding variations required to reconstruct the data from approximations. Both approximations and details are represented at a reduced rate, by a factor of 2 for each dimension. However, the combination of the forward and inverse transformations is exact, allowing perfect reconstruction. Figure 1 illustrates a resulting 3D decomposition of volumetric data (left) in red. A fraction $\left(1 / 2^{3}\right)$ of the decomposed data in red (right) corresponds to an approximation of the initial data at a lower resolution. Remaining details (the seven transparent subbands) are stored to allow a perfectly revertible transformation. The same decomposition can be iteratively applied to the lower resolutions, generating a hierarchy of embedded approximation levels.

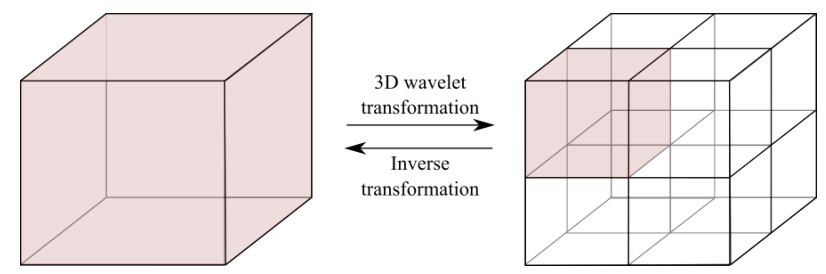

Figure 1: Left: 3D decomposition of a volumetric data (red). Right: decomposed data, consisting of a data approximation (red) and 7 detail subbands (transparent).

HexaShrink combines five different decomposition types. For mesh geometry, a nonlinear morphological wavelet manages a visual preservation of the fault network across the resolutions. Continuous properties such as porosity and permeability are decomposed with the separable 3D wavelet used by the image compression standard JPEG2000 (Acharya and Tsai, 2005). More details are provided in (Peyrot et al., 2019, 2016). Note that such discrete wavelets have already been used to scale continuous properties up (Pazhoohesh et al., 2006; Rasaei and Sahimi, 2008), with promising results. Franc et al. (2017) recently dealt with similarities shared between upscaling and multiscale methods.

A grid has been modeled to test simulation performance on the hierarchical resolutions. It includes three faults, whose surface directions do not follow grid orientations, as illustrated by the left-most grid on Figure 2. The model is 3400 meters deep, with dimensions $220 \times 220 \times 30$ meters. The originalscale model size is $128 \times 128 \times 32$ cells, with a fine-scale cell size of $1.70 \times 1.70 \times 0.95 \mathrm{~m}^{3}$. Power-of-two dimensions have been intentionally used to simplify scale changes and comparisons. Its properties, porosity and permeability, have been simulated using reference histograms and variograms. They are based on the Tarbert formation from the SPE-10 model (Christie and Blunt, 2001). It corresponds to a near shore environment.

HexaShrink can perform up to 5 levels of decomposition with this model. Only the first three levels are represented on the geometry in Figure 2. The faults remain visually coherent across resolutions. The uniformly reduced number of cells across resolutions is detailed in Table 1. The corresponding wavelet decompositions on porosity and permeability are represented in the first two columns of Figure 3.

A two-phase flow simulation is performed on the reservoir grid corresponding to a quarter of five spot model. Two wells, one injector and one producer, are distant from about 300 meters. They are placed 


\begin{tabular}{|c|c|r|}
\hline Resolution & Fine scale model size & Total cell number \\
\hline 0 & $128 \times 128 \times 32$ & 524288 \\
-1 & $64 \times 64 \times 16$ & 65536 \\
-2 & $32 \times 32 \times 8$ & 8192 \\
-3 & $16 \times 16 \times 4$ & 1024 \\
-4 & $8 \times 8 \times 2$ & 128 \\
-5 & $4 \times 4 \times 1$ & 16 \\
\hline
\end{tabular}

Table 1: Dimensions of the initial mesh and meshes generated by HexaShrink.

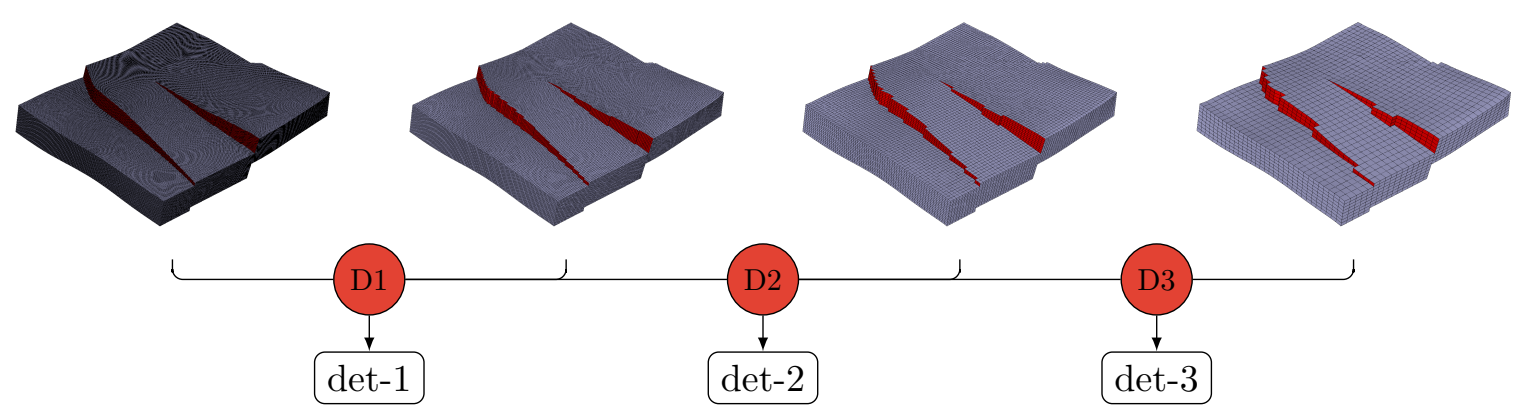

Figure 2: Performing successively 3 decomposition levels $(D i)$ ) on an initial grid (on the left) allows to generate, for each level, approximation data (a grid at lower resolution) and details (det-i). Red surfaces highlight the faults, conserved across resolutions.

at opposite places on the surface mesh diagonal: the injector is localized on the lower corner while the producer is set on the highest point of the reservoir. Simulations are performed on the different mesh resolutions provided by HexaShrink. Results are represented on the two last columns of Figure 2. The fourth column is similar to the third, with water saturation thresholded below 0.50 .

\section{Results}

Simulation parameters are fixed to compare the results obtained on the several decompositions. The injector well pressure is constant at 300 bars, while the producer rate is constant at $300 \mathrm{~m}^{3}$ per day. This preliminary study focuses on simulation results at the whole grid scale. We for instance observe the saturation property, and especially the progress front of water injection displayed in the two last columns of Figure 3. Its advance is impacted by the properties of the mesh, as well by its structure, notably the fault network. Taking the original grid as the reference, we compare the evolution of the progress front across lower resolutions. Sufficient similarities in the outcome can substantiate the interest of a compressed format providing a direct access to lower resolutions.

After 76 simulation days, the injected water (water-saturated at 0.50, see Figure 3, column 4) reaches the producer in the original grid. Its localization is symbolized by the red dashed line. For lower resolutions, water has not attained the producer yet. However, the remaining distance is relatively short for resolutions -1 and -2 . It becomes significant after resolution -3 . Similar behaviors in the global shape of the progress front are identified. For instance, water accumulates at specific areas against the faults, whatever the resolution considered. Considering the drastic computational gains at resolutions -1 and -2 (with an 8- and 64-fold cell reduction respectively), such scales could be exploited with the purpose 


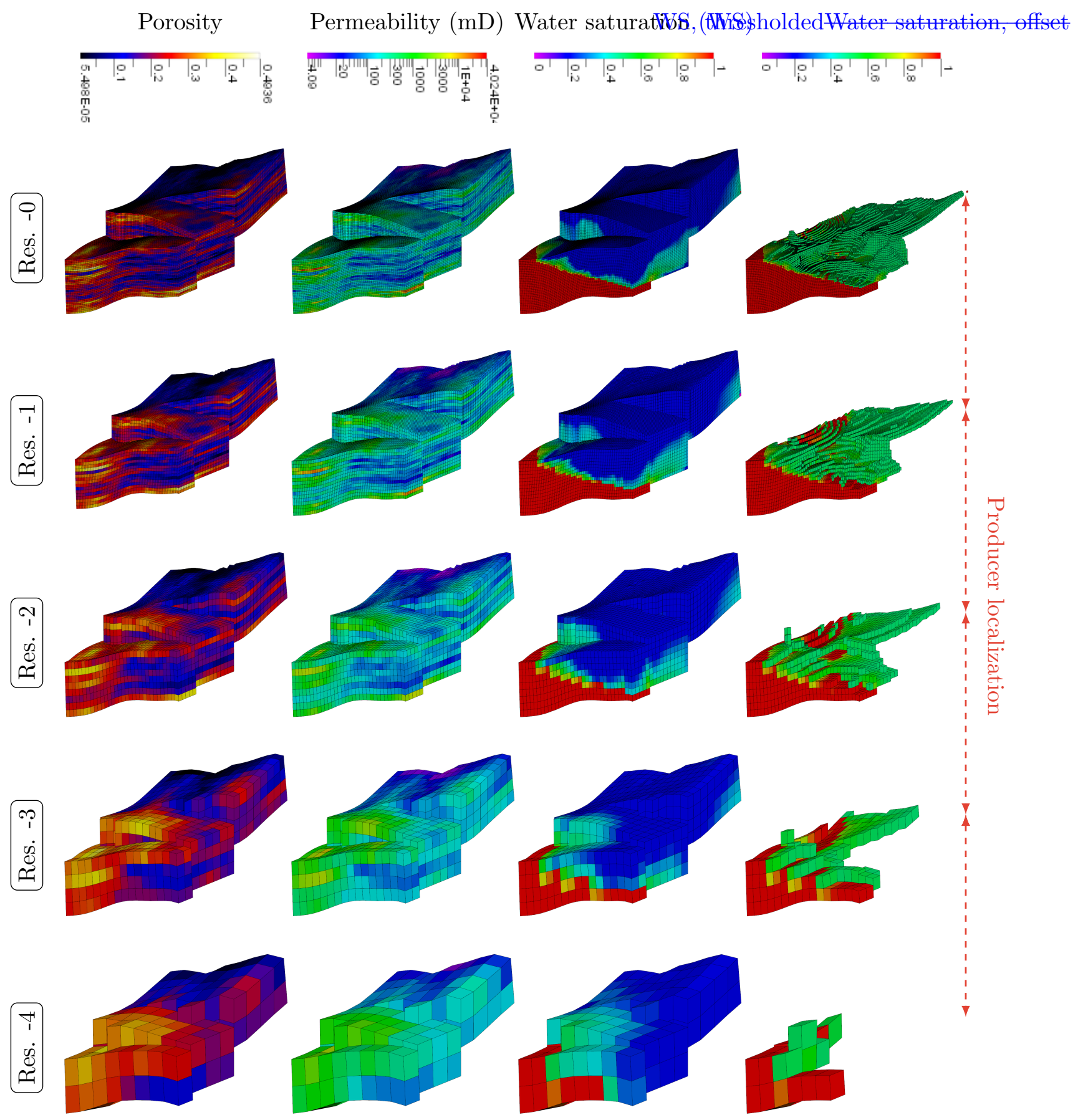

Figure 3: From left to right: meshes with physical attributes (porosity, permeability), and water saturation after 76 simulation days. From top to bottom: original mesh and four levels of decomposition generated with Hexashrink. The red dashed line marks the producer well localization. It is reached for finer resolutions $(-1$ and -2$)$, and exhibits an increasing delay for lower resolutions $(-3$ and -4$)$. 
of accelerated, approximated simulations.

\section{Conclusions}

A two-phase flow simulation has been successfully run on multiresolution meshes generated by HexaShrink, a fully reversible decomposition/compression. Using its original scheme, HexaShrink manages to store and to progressively decompress meshes at increasing levels of details. Visually, the structural coherency of the meshes, especially its fault network, are preserved across resolutions. This preservation is tested in the simulation context, studying the progress front of water injection, affected by the structure of the grid. Using the original resolution as a reference, the impact of resolution reduction is evaluated. If extreme shrinkage of structural details reduce simulation quality, the first two resolution results exhibit similarity with the reference one. As a result, owing to the storage and transfer gain allowed by compression, potential benefits in faster approximate simulations, encourage us to pursue the possibility of embedded grids at different scales with reduced upscaling and upgriding overhead.

\section{References}

Acharya, T. and Tsai, P.-S. (2005). JPEG2000 Standard for Image Compression. Concepts, Algorithms and VLSI Architectures. Wiley-Interscience. 2

Bouard, L., Duval, L., Payan, F., and Antonini, M. (2018). Décomposition multi-échelles de maillages 3D hexaédriques dans le domaine des géosciences. Étude des performances en compression sans pertes. 1

Christie, M. and Blunt, M. (2001). Tenth SPE comparative solution project: A comparison of upscaling techniques. In SPE Reservoir Simulation Symposium. Society of Petroleum Engineers. 2

Franc, J., Debenest, G., Jeannin, L., and Masson, R. (2017). Comparison between multiscale finite volume methods in reservoir engineering. In Proc. EAGE Conf. Tech. Exhib. 2

Mallat, S. (2009). A wavelet tour of signal processing: the sparse way. Academic Press, San Diego, CA, USA, 3rd edition. 2

Pazhoohesh, E., Hamzehpour, H., and Sahimi, M. (2006). Numerical simulation of ac conduction in three-dimensional heterogeneous materials. Phys. Rev. B, 73(17). 2

Peyrot, J.-L., Duval, L., Payan, F., Bouard, L., Chizat, L., Schneider, S., and Antonini, M. (2019). HexaShrink, an exact scalable framework for hexahedral meshes with attributes and discontinuities: multiresolution rendering and storage of geoscience models. Computat. Geosci. (UNDER SECOND REVIEW). 1, 2

Peyrot, J.-L., Duval, L., Schneider, S., Payan, F., and Antonini, M. (2016). (H)exashrink: Multiresolution compression of large structured hexahedral meshes with discontinuities in geosciences. In Proc. Int. Conf. Image Process., pages 1101-1105. 1, 2

Ponting, D. K. (1989). Corner point geometry in reservoir simulation. In ECMOR I - 1st European Conference on the Mathematics of Oil Recovery. EAGE Publications BV. 1

Rasaei, M. and Sahimi, M. (2008). Upscaling and simulation of waterflooding in heterogeneous reservoirs using wavelet transformations: Application to the SPE-10 model. 72(3):311-338. 2 